\title{
Lasing action in microdroplets modulated by interfacial molecular forces
}

\author{
Zhen Qiao, ${ }^{a}$ Xuerui Gong, ${ }^{\text {a Peng Guan, }}{ }^{\mathrm{b}}$ Zhiyi Yuan, ${ }^{\text {a }}$ Shilun Feng, ${ }^{\mathrm{c}}$ Yiyu Zhang, ${ }^{\mathrm{a}}$ Munho Kim $\odot,{ }^{\mathrm{a}}$ Guo-En Chang $\odot,{ }^{\mathrm{d}}$ \\ and Yu-Cheng Chen $\oplus^{\mathrm{a}, \mathrm{e}, *}$ \\ aNanyang Technological University, School of Electrical and Electronic Engineering, Singapore \\ ${ }^{b}$ Shanghai Jiao Tong University, School of Electronic Information and Electrical Engineering, Shanghai, China \\ ${ }^{\circ}$ Chinese Academy of Sciences, Shanghai Institute of Microsystem and Information Technology, \\ State Key Laboratory of Transducer Technology, Shanghai, China \\ ${ }^{d}$ National Chung Cheng University, Department of Mechanical Engineering, Minhsiung, Chiayi, Taiwan

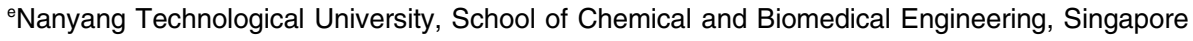

\begin{abstract}
Liquid droplets offer a great number of opportunities in biochemical and physical research studies in which droplet-based microlasers have come into play over the past decade. While the recent emergence of droplet lasers has demonstrated their powerful capabilities in amplifying subtle molecular changes inside the cavity, the optical interactions between droplet resonators and an interface remain unclear. We revealed the underlying mechanism of droplet lasers when interacting with a droplet-solid interface and explored its correlation with intermolecular forces. A vertically oriented oscillation mode-arc-like mode-was discovered, where the number of lasing modes and their $Q$-factors increase with the strength of interfacial hydrophobicity. Both experimental and theoretical results demonstrated that hydrophobicity characterized by contact angle and interfacial tension plays a significant role in the geometry of droplet cavity and laser mode characteristics. Finally, we demonstrated how tiny forces induced by proteins and peptides could strongly modulate the lasing output in droplet resonators. Our findings illustrate the potential of exploiting optical resonators to amplify intermolecular force changes, providing comprehensive insights into lasing actions modulated by interfaces and applications in biophysics.
\end{abstract}

Keywords: microlaser; droplet resonator; intermolecular forces; biointerface; lasing action; hydrophobicity.

Received Oct. 26, 2020; revised manuscript received Dec. 6, 2020; accepted for publication Dec. 23, 2020; published online Jan. 27, 2021.

(C) The Authors. Published by SPIE and CLP under a Creative Commons Attribution 4.0 Unported License. Distribution or reproduction of this work in whole or in part requires full attribution of the original publication, including its DOI.

[DOI: 10.1117/1.AP.3.1.016003]

\section{Introduction}

Microlasers have emerged as promising tools in modern science and technology. ${ }^{1-18}$ Typical types of microcavities include Fabry-Perot cavities and whispering gallery mode (WGM)based cavities, which are represented by ring resonators, microdisks, and droplet resonators. ${ }^{9}$ Over the past decade, dropletbased microlasers have attracted great attention for their biocompatibility, low scattering losses, and easy fabrication. ${ }^{11-17}$ In general, droplet resonators are formed by supporting droplets with solid surfaces. In the process of liquid interacting with a solid surface, intermolecular forces at the interface mainly

*Address all correspondence to Yu-Cheng Chen, yucchen@ntu.edu.sg determine the mechanical equilibrium of molecules. ${ }^{19}$ In particular, hydrophobic forces at liquid-solid interfaces play a vital role in numerous physical dynamics and biological systems. ${ }^{20-31}$

Recent advances have been achieved using droplets to form microlasers for the applications of intracavity-based sensors and optoelectronic devices. ${ }^{11-17}$ When a spherical droplet interacts with an interface to form a contact angle, the dramatic mechanical changes at the interface should play a significant role in its optical oscillation. ${ }^{32,33}$ At present, most droplet resonators are defined by horizontally oriented WGM oscillation. By supporting a microdroplet on a super-hydrophobic solid surface to form an obtuse contact angle, WGM lasing was realized in droplets through light oscillations along the horizontal equators. ${ }^{12,13}$ Several groups have also demonstrated WGM lasing in mirror-supported 

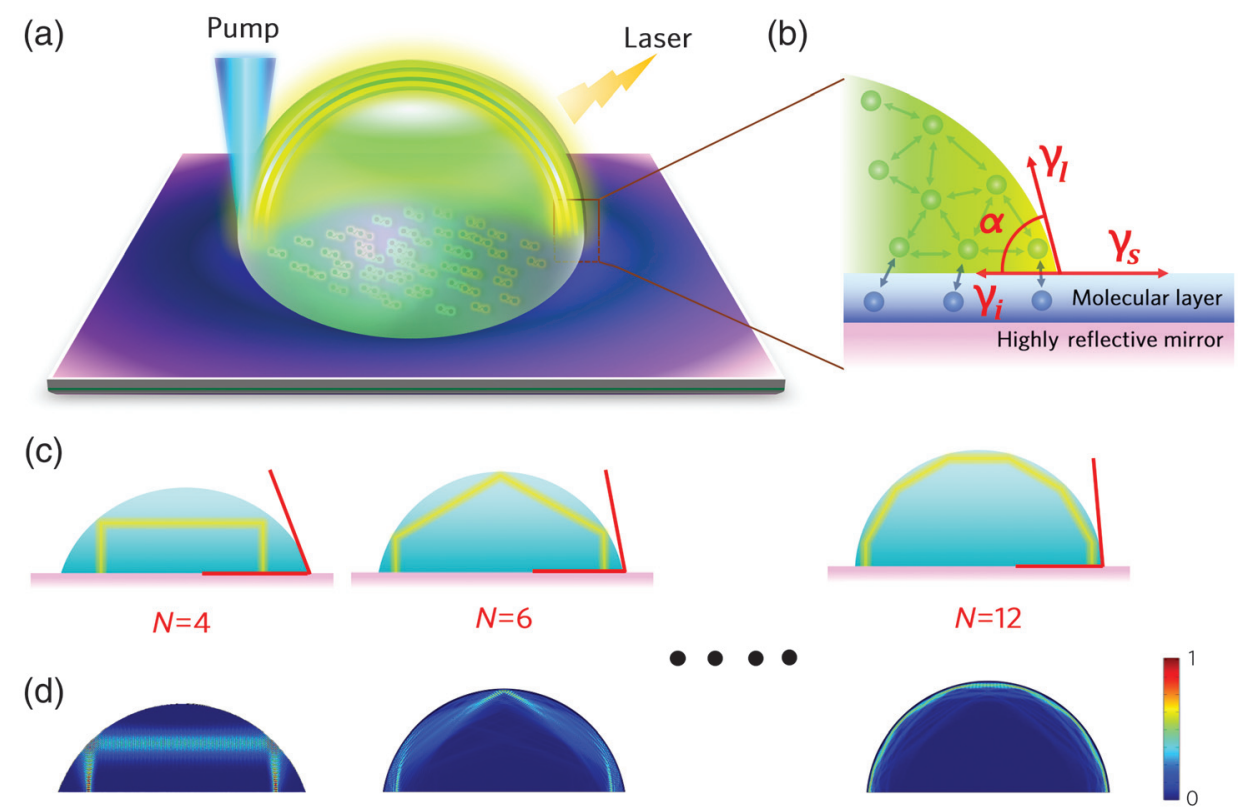

Fig. 1 Concept and principle of droplet lasers hosting AL modes based on different hydrophobic forces at droplet-solid interfaces. (a) Schematic of a droplet laser hosting AL modes. A dye-doped droplet is formed on a highly reflective dielectric mirror. AL modes will oscillate from strong reflections between the mirror and the droplet-air interface. (b) Enlarged schematic of the dropletsolid interface in (a). A molecular layer is deposited between the mirror and the droplet. The droplet-solid interfacial tension $\gamma_{i}$, the solid surface tension $\gamma_{s}$, and the droplet surface tension

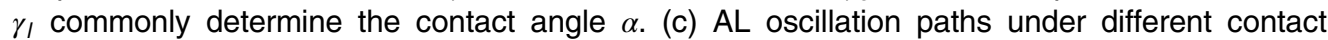
angles. It is worth noting that AL modes will follow the paths corresponding to segments of $N$-sided polygons. As the contact angle increases, AL modes with a larger $N$ will be excited. (d) The simulated normalized electric field distributions of AL modes under different contact angles in (c).

droplets with acute contact angles, where the WGM oscillates along the bottom circumference of droplets. ${ }^{14-17}$ Although many proof-of-principles exist, the correlation between the lasing actions of droplet resonators and the interfacial hydrophobicity remains unrevealed. Therefore, revealing the fundamental correlation between droplet lasers and droplet-solid interfaces may open new insights into microlasers.

In this work, we revealed the underlying mechanism of interfacial hydrophobicity modulating lasing action in microdroplets and subsequently employed biointerfaces to modulate the laser emission of droplet resonators. Lasing action of droplet resonators with different molecular hydrophobicity at droplet-solid interfaces were explored in which a vertically oriented oscillation mode-arc-like (AL) mode-was discovered. AL modes that resonate along the droplet surface between its two ends were demonstrated to be strongly influenced by the intermolecular forces at the droplet-solid interface (Fig. 1). With the increasing of interfacial tension (higher hydrophobicity and larger droplet contact angle), more AL modes with higher $Q$-factors will be excited, resulting in significant lasing output. Finally, we showed how biomolecules alter the hydrophobic forces and strongly modulate the laser modes of droplet resonators. This work reports the important modulating mechanism in droplet resonators, which may open new perspectives for fundamental studies in interface physics as well as the applications in biomechanical detection.

\section{Materials and Methods}

\subsection{Fabrication of Droplet Resonators}

For all the results shown in Figs. 2-4, the droplets were prepared by mixing DI water/glycerol (volume ratio $=1: 1$ ). The purpose of mixing water and glycerol is to prevent droplets from evaporation. To confirm this effect, we compared the size changes of a droplet over periods of time with and without adding glycerol in Fig. S1 in the Supplementary Material. No obvious changes of the water/glycerol droplets were observed in $7 \mathrm{~h}$; in contrast, the size of the water droplets shrank dramatically in 20 min due to evaporation. The slow evaporation of the water/glycerol mixture can be attributed to the hydroscopic nature of glycerol. ${ }^{37}$ For the preparation of lasing droplets, $2 \mathrm{mM}(1 \mathrm{mM}=$ $1 \mathrm{mmol} / \mathrm{L}$ ) fluorescein sodium salt (FITC, Sigma-Aldrich \#F6377) solution was prepared and printed on a dielectric mirror (customized by Evaporation Coatings Inc.) or a glass slide using an inkjet printer (Fig. S2 in the Supplementary Material). The hydrophobicity of the mirror in Fig. 4 was tuned by treating the mirror with different ratios of hydrophobic reagent/ethanol solutions. For the recording of molecular hydrophobicity in Fig. 5, the droplet resonators with different biomolecular concentrations were fabricated on a dielectric mirror using an inkjet printer (Fig. S2 in the Supplementary Material). First, molecular solutions with varying concentrations of bovine serum 
(a)

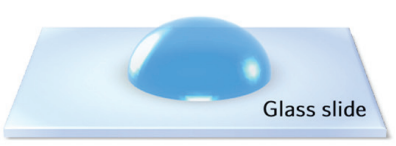

(b)

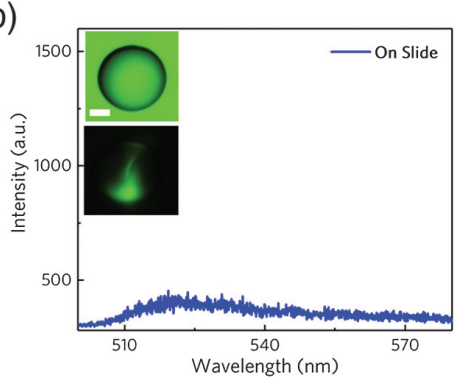

(g)

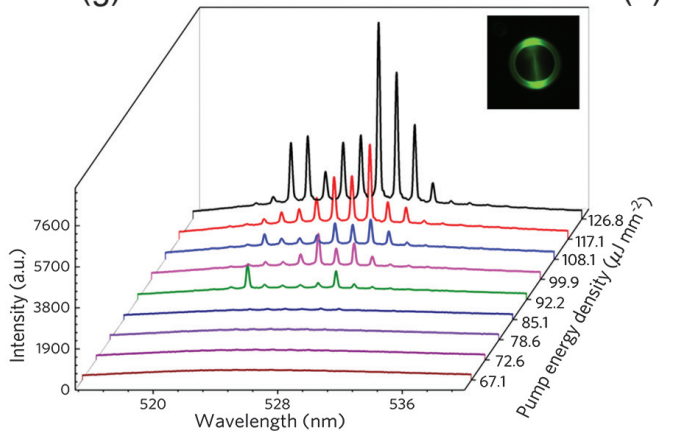

(c)

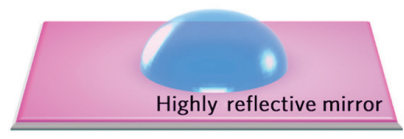

(d)

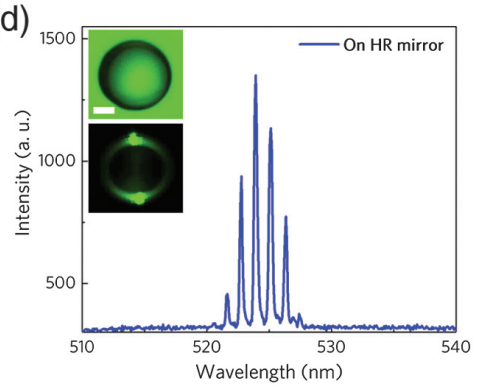

(h)

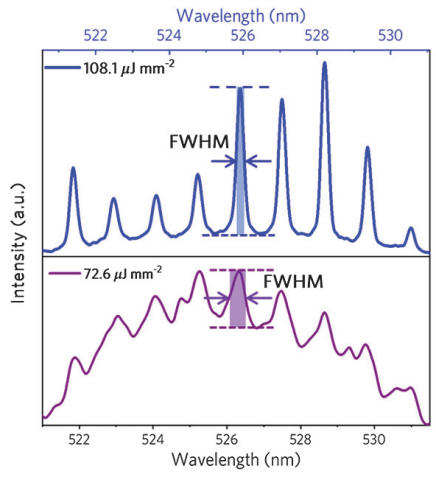

(e)

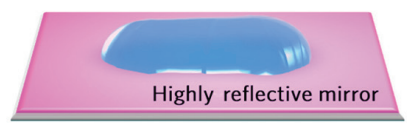

(f)

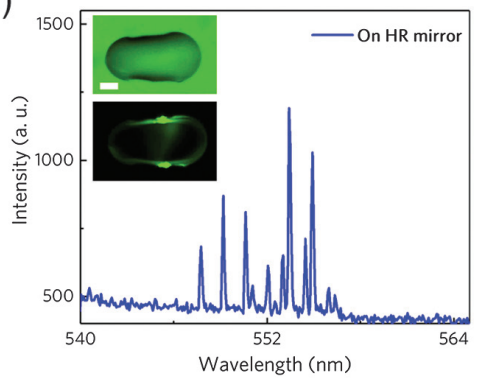

(i)

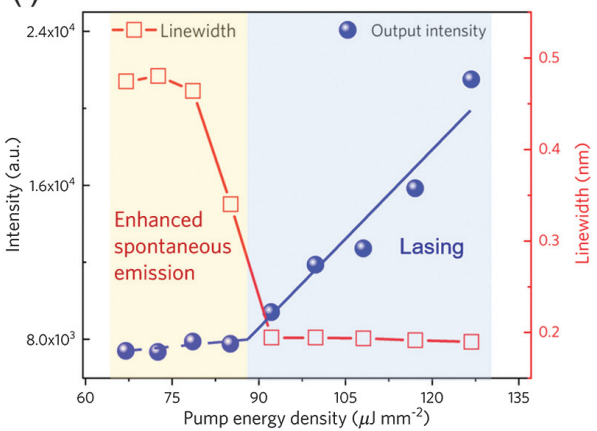

Fig. 2 Demonstration of lasing action. (a) Schematic of a round droplet on a glass slide. (b) The optical spectrum of the droplet on a glass slide showing no laser emission. Inset: top-view profile of a round droplet on a glass slide (top) and the optical image after pumping (below). (c) Schematic of a round droplet on a highly reflective mirror. (d) The optical spectrum of the round droplet on the mirror showing laser emission. Inset: top-view profile of a round droplet on a mirror (top) and the optical image after pumping (below). (e) Schematic of a "deformed" droplet on a highly reflective mirror. (f) The optical spectrum of the "deformed" droplet showing laser emission. Insets: top-view profile of the "deformed" droplet (top) and the optical image after pumping (below). Scale bars in insets: $20 \mu \mathrm{m}$. (g) Optical spectra of a droplet resonator with increasing pump energy densities. Inset: optical image of the droplet laser. (h) Enlarged spectra of the droplet resonator under the pump energy of $72.6 \mu \mathrm{J} / \mathrm{mm}^{2}$ (below threshold) and $108.1 \mu \mathrm{J} / \mathrm{mm}^{2}$ (above threshold). FWHM: full width at half-maximum. (i) Spectrally integrated output intensity and linewidth as a function of pump energy density [extracted from (g)]. FITC concentration: $2 \mathrm{mM}$.

albumin (BSA) (Supelco \#P5369), insulin (Sigma Aldrich \#I0908), and V5 peptide (Sigma Aldrich \#V7754) were prepared by dissolving specific amounts of biomolecules into buffer solutions. For the BSA and V5, phosphate-buffered saline (PBS) buffer was used as the solvent. For the insulin, triethylammonium bicarbonate buffer (Sigma Aldrich \#T7408) was used as the solvent. Then the protein/peptide solution was mixed with glycerol (volume ratio $=3: 1$ ) and FITC to prepare a final solution with $4 \mathrm{mM}$ FITC. The purpose of decreasing the volume ratio of glycerol is to reduce the hindrance of contact angle changes induced by the droplet viscosity. In Figs. 4 and 5, the droplets were fabricated using the same printer and program, thus the volumes of the droplets with different contact angles were close (about $68.5 \mathrm{pL}$ ). The dielectric mirror used in Fig. 5 was previously treated by octadecyl trichlorosilane (SigmaAldrich \#104817) to form a hydrophobic surface.

\subsection{Optical System Setup}

The schematic of the optical system can be seen in Fig. S2(b) in the Supplementary Material. Optical pumping was achieved by a pulsed ns-laser (EKSPLA PS8001DR) integrated with an optical parametric oscillator (repetition rate: $50 \mathrm{~Hz}$; pulse duration: $5 \mathrm{~ns}$ ). According to the absorption spectrum of FITC, $479 \mathrm{~nm}$ was selected as the pumping wavelength. An upright microscopic system (Nikon Ni2) with a $10 \times 0.3$ NA objective was used to focus the pump light onto the droplets and was also used to collect the emissions from the droplets. The beam diameter at the objective focal plane was measured to be $40 \mu \mathrm{m}$. The collected emissions from droplets were separated by a beam splitter and incident into a charge-coupled device (CCD) camera (Newton 970 EMCCD) and imaging spectrometer (Andor Kymera 328i). The side-view droplet profiles were 
(a)

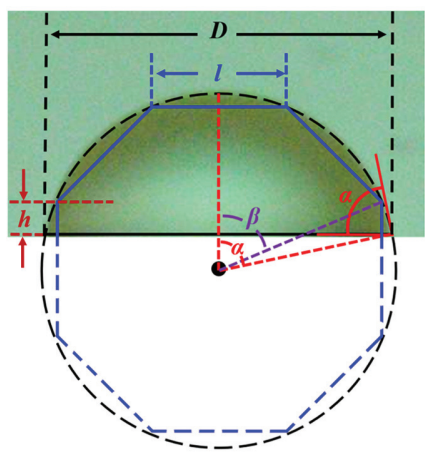

(b)

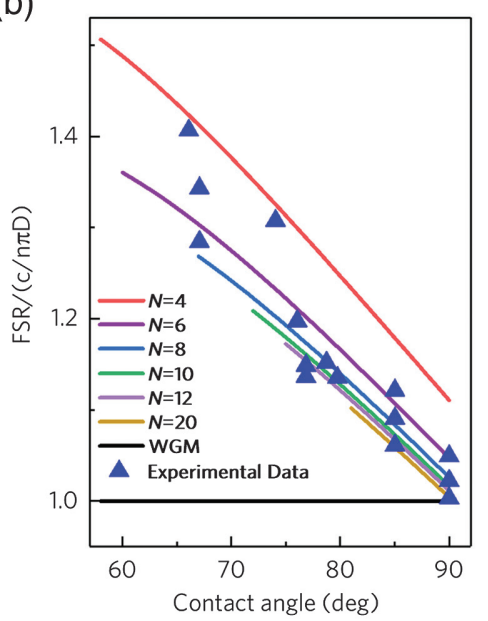

(c)

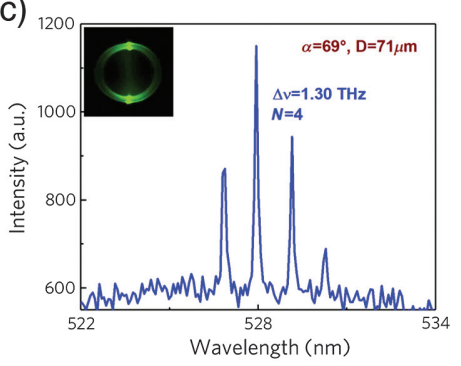

(d)

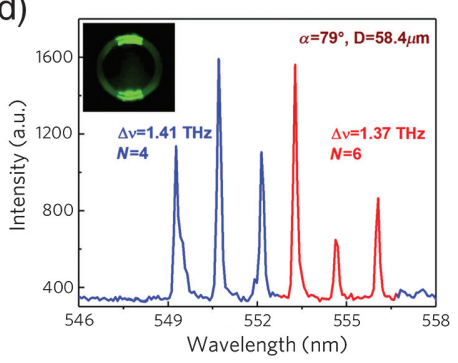

(e)

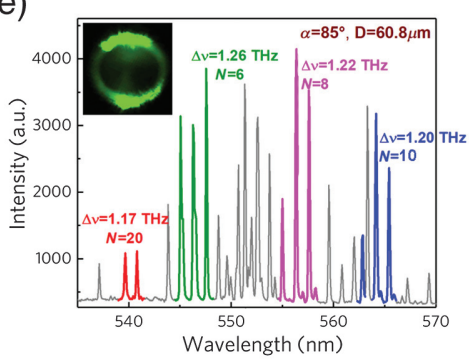

(f)

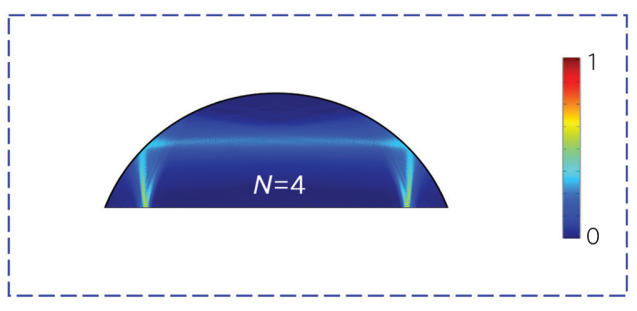

(g)

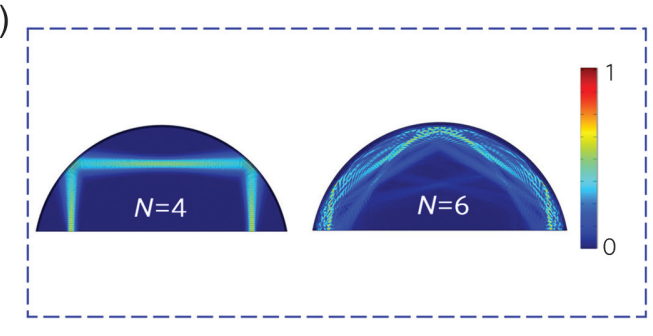

(h)

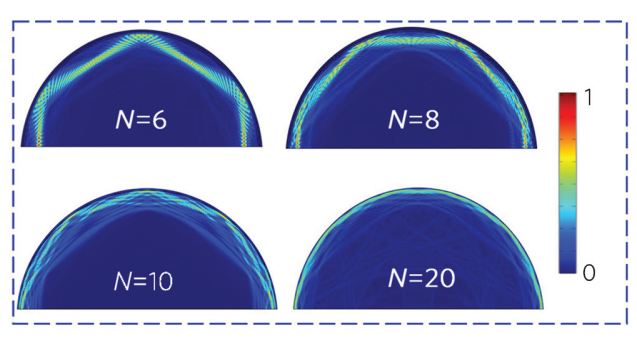

Fig. 3 Characterization of the AL modes through FSRs. (a) Side-view profile of a droplet. $\alpha$, contact angle. $D$, diameter. Black dot circle, the complete circle containing the droplet profile. Blue line, an $\mathrm{N}$-sided polygon containing the AL mode oscillation path (solid blue line). I, side length of an $N$-sided polygon. $h$, length of the two vertical short sides. (b) Measured FSRs (blue triangles) and the theoretical lines calculated by considering WGM (black line) and AL modes (colored lines). (c)-(e) The optical spectra of droplet resonators with the contact angles of (c) 69 deg, (d) 79 deg, and (e) 85 deg, respectively. Insets: CCD images of lasing emissions from individual droplets. (f) Simulated electric field distribution of the AL mode with $N=4$ calculated through the FSR in (c). (g) Simulated electric field distributions of the AL modes with $N=4$ and $N=6$ calculated through the FSRs in (d). (h) Simulated electric field distributions of the AL modes with $N=6$, $N=8, N=10$, and $N=20$ calculated through the FSRs in (e). FITC concentration: $2 \mathrm{mM}$. Pump energy density: $78 \mu \mathrm{J} / \mathrm{mm}^{2}$.

recorded by a color CCD (Andor Zyla SCMOS) mounted on an inverted microscopic system (Nikon Ti2) with $20 \times 0.4$ NA objective.

\subsection{Simulations}

The electric field distributions of AL modes and WGM and their $Q$-factors were calculated with the finite element method using COMSOL Multiphysics software. The eigenfrequency study was applied in the electromagnetic waves, frequency-domain interface within the wave optics modules. For AL modes, a 2D model was employed. For WGM, a 2D axisymmetric model was employed. The geometries of the droplet resonators were set to be parts of perfect rounds, and the refractive index (RI) was 1.41 . For the $Q$-factor calculations, a bottom mirror with a reflectivity of $\sim 99.995 \%$ was considered, which introduced $\sim 0.01 \%$ losses for AL modes and thereby limited the $Q$-factors of AL modes up to the $\sim 10^{7}$ level. A bottom distributed Bragg reflector (DBR) was composed of 24 dielectric layers alternating between high-RI (2.32) and low-RI (1.38) layers. The thickness of the high-RI layers was $58.2 \mathrm{~nm}$; and the thickness of the low-RI layers was $97.8 \mathrm{~nm}$. The $Q$-factors under each contact angle were the maximum values selected from 100 solutions around 540-nm wavelength obtained by COMSOL Multiphysics software.

\section{Results}

\subsection{Concept and Principle of Droplet Resonators Hosting AL Modes}

Figure 1 presents the fundamental concept and principle of droplet resonators hosting AL modes based on different hydrophobic forces at droplet-solid interfaces. A droplet doped with 
(a)
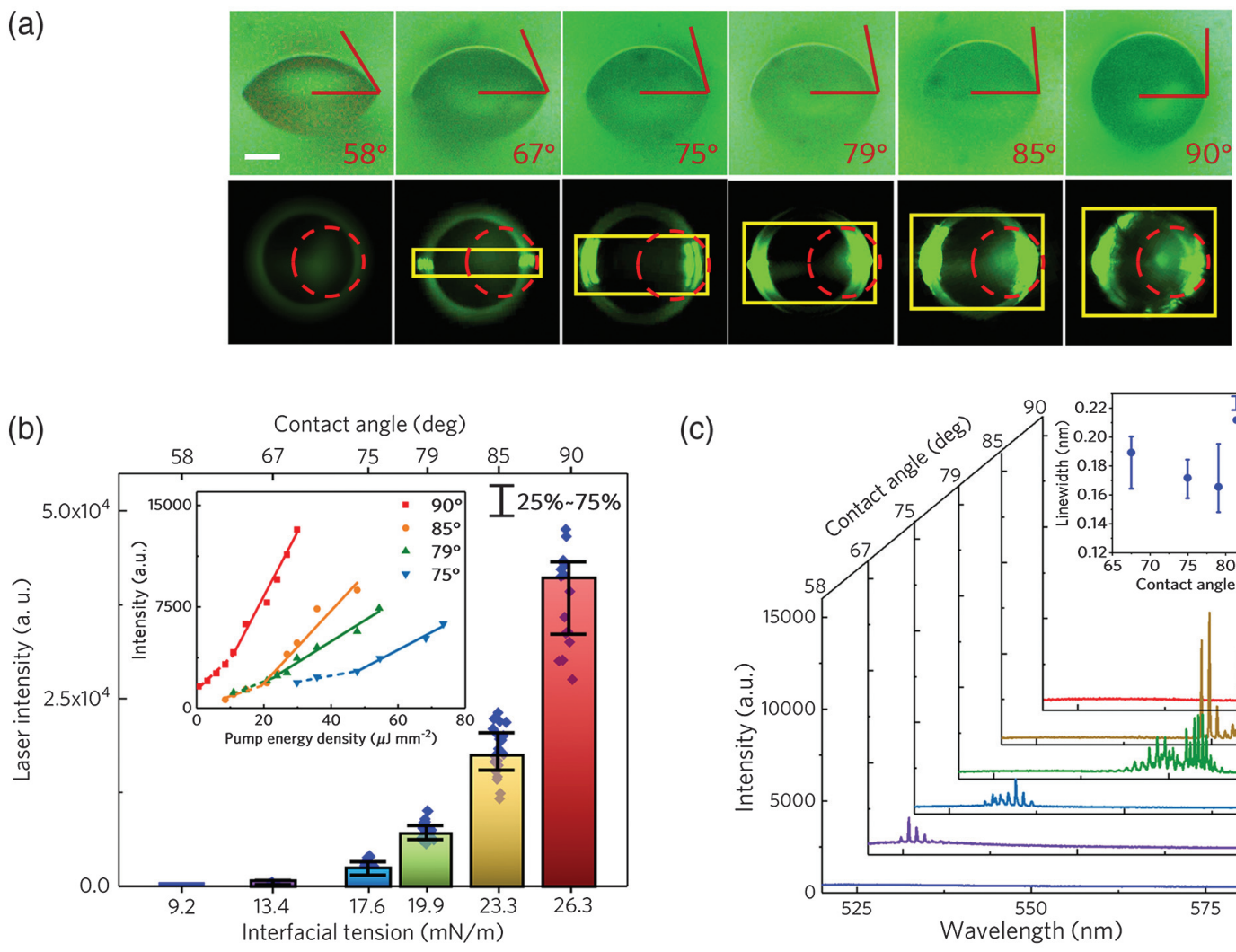

(c)

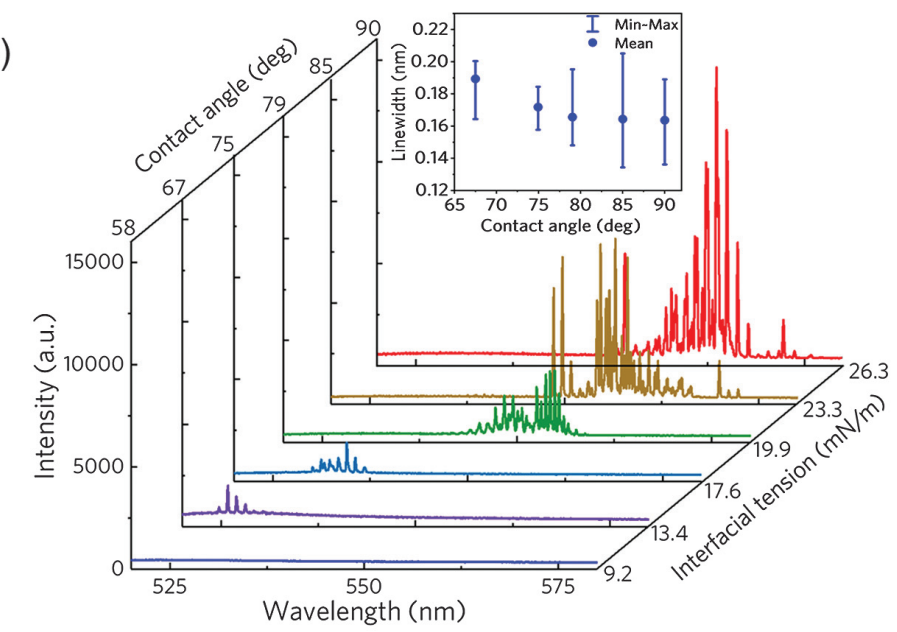

(d)

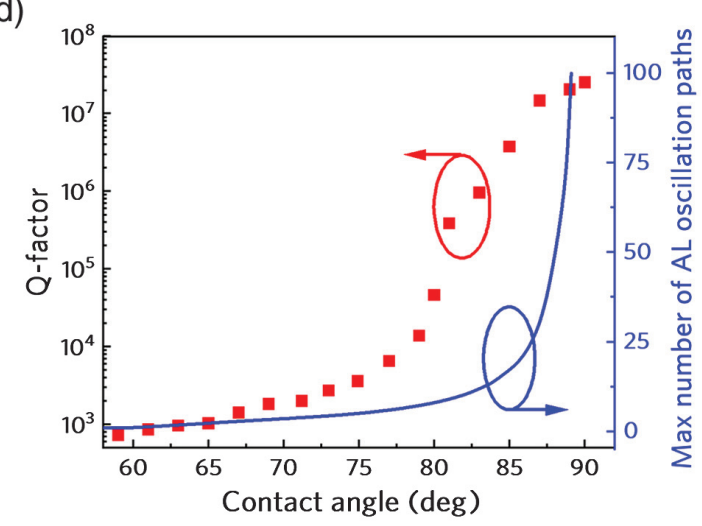

(e)

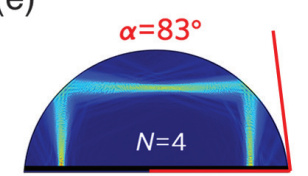

$Q=1.91 \times 10^{3}$

(f)

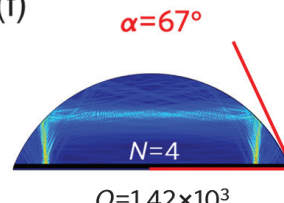

$Q=1.42 \times 10^{3}$

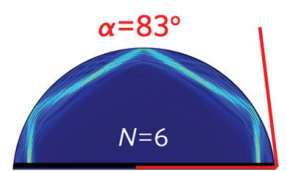

$\mathrm{Q}=5.94 \times 10^{5}$

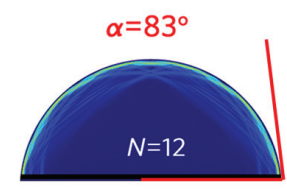

$Q=7.61 \times 10^{5}$

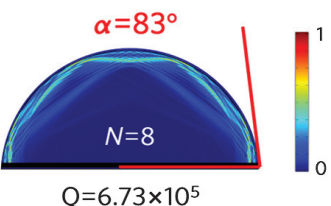

$Q=6.73 \times 10^{5}$

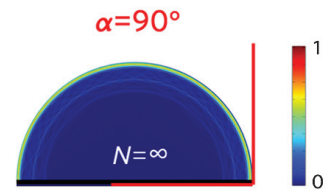

$Q=2.31 \times 10^{7}$

Fig. 4 Lasing action of droplet lasers under different hydrophobicity. (a) Top: side-view profiles of droplets with varying contact angles. Below: optical images of the droplets after pumping. Yellow boxes, the laser emission regions. Red dashed circle in the CCD images represents excitation pump regions. Scale bar: $20 \mu \mathrm{m}$. (b) Statistics of spectrally integrated laser outputs at different interfacial tensions and contact angles under the same pump energy density. Inset: spectrally integrated laser outputs as a function of pump energy density at contact angles of $75 \mathrm{deg}, 79 \mathrm{deg}, 85 \mathrm{deg}$, and $90 \mathrm{deg}$. (c) Optical spectra of droplet resonators with different interfacial tensions. Inset: laser linewidths under different contact angles. (d) Calculated $Q$-factors and the maximum number of AL oscillation paths as a function of contact angle. (e) Simulated electric field distributions and $Q$-factors of the AL modes with $N=4,6$, and 8 under the same contact angle of $83 \mathrm{deg}$. (f) Simulated electric field distributions and $Q$-factors of the AL modes with $N=4,12$, and $\infty$ for respective contact angles of $67 \mathrm{deg}, 83 \mathrm{deg}$, and $90 \mathrm{deg}$. FITC concentration: $2 \mathrm{mM}$. Pump energy density: $78 \mu \mathrm{J} / \mathrm{mm}^{2}$. Calculation parameters: volume of droplets $=68.5 \mathrm{pL}$; refractive index $=1.41$. Interfacial tension calculations: ${ }^{34-36}$ refer to Sec. 2 in the Supplementary Material. 
(a)

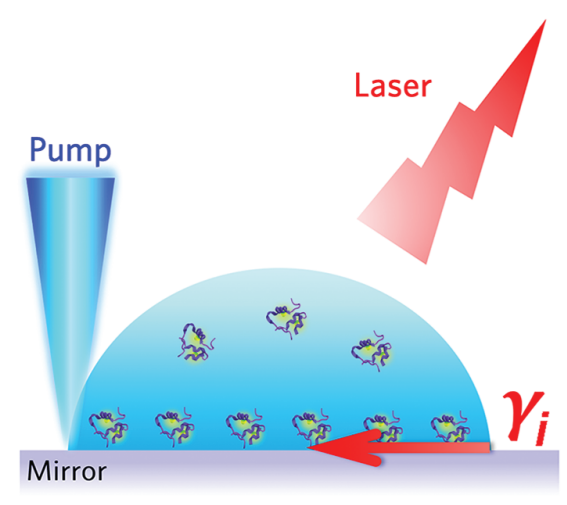

Low concentration

Droplet resonators with biomolecules

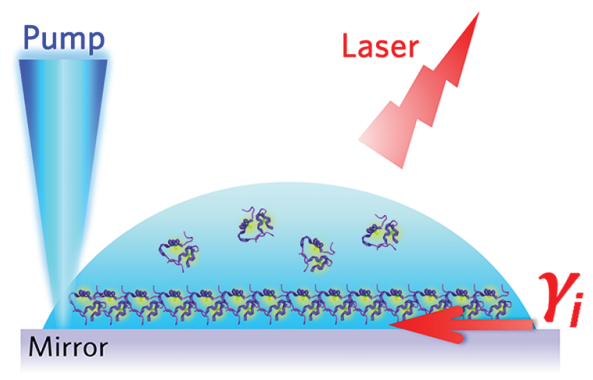

High concentration

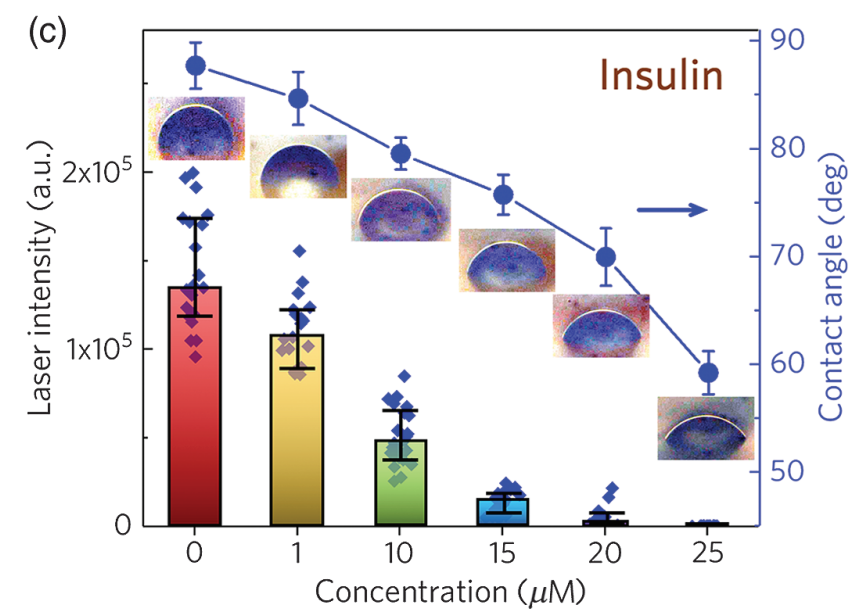

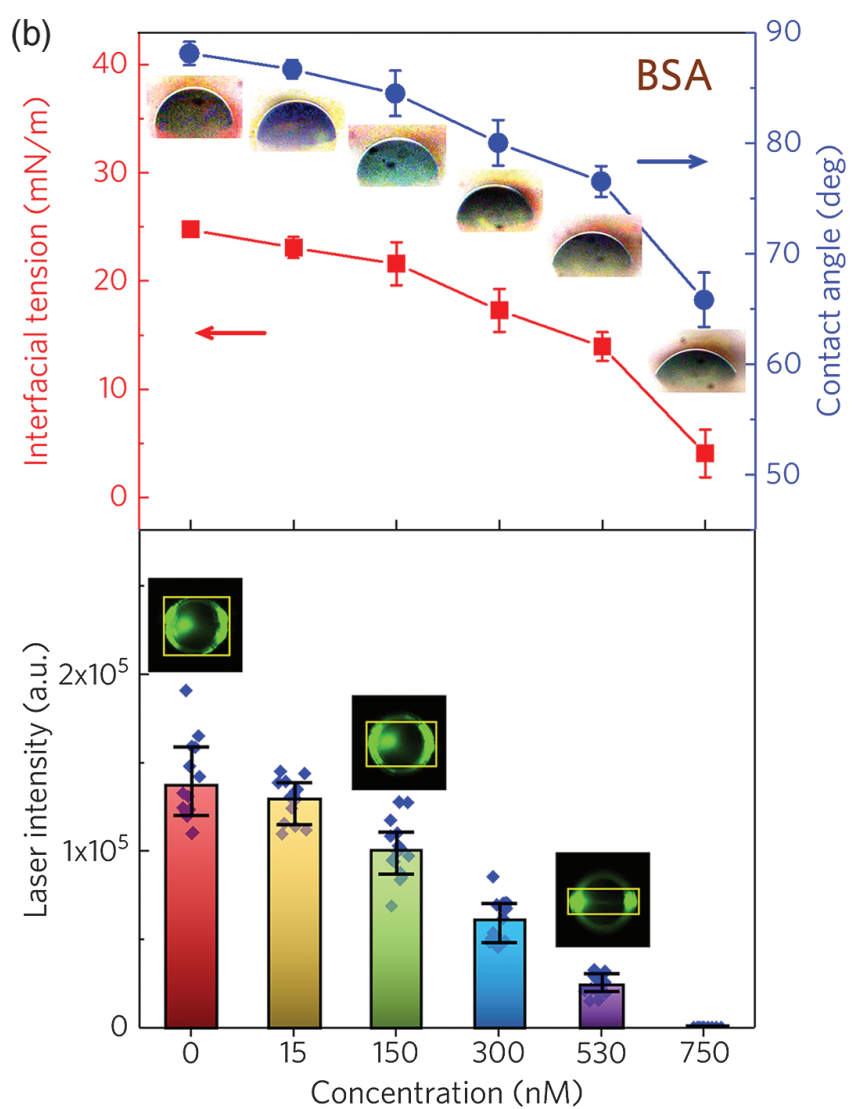

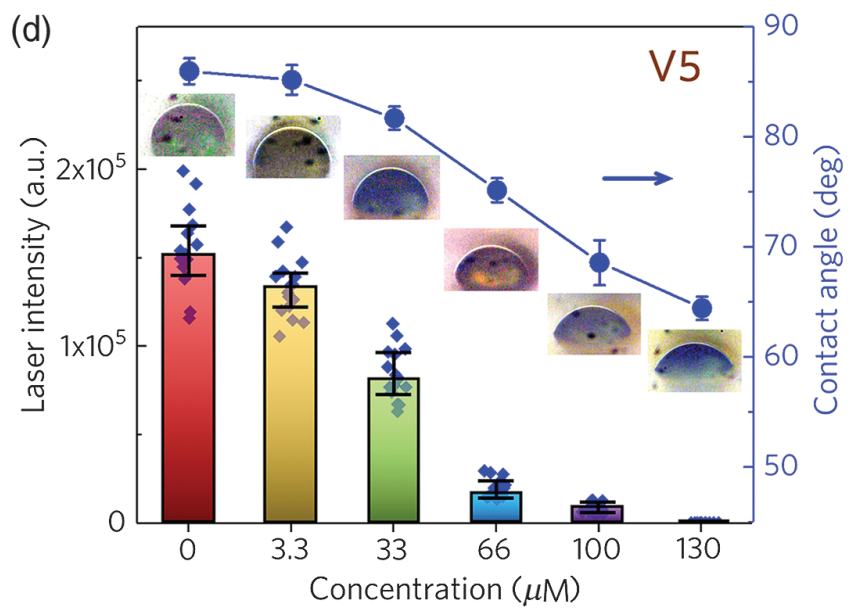

Fig. 5 Modulating laser emission with intermolecular forces at the biointerface. (a) Schematic of biointerface modulating laser emissions based on biomolecular adsorptions with different concentrations. Higher concentration of biomolecules leads to smaller interfacial tension $\gamma_{i}$ and lower laser intensity. (b) Interfacial tensions (top), contact angles (top), and spectrally integrated laser outputs (bottom) as a function of BSA concentration. (c), (d) Contact angles and spectrally integrated laser outputs as a function of different biomolecular concentration, including (c) insulin and (d) V5 peptide. Insets in (b) (top), (c), and (d): side views of droplets with different biomolecular concentrations. Inset in (b) (bottom): CCD images of lasing emissions under different concentrations, where the emission regions are marked with yellow boxes. FITC concentration: $4 \mathrm{mM}$. Pump energy density: $36 \mu \mathrm{J} / \mathrm{mm}^{2}$. Interfacial tension calculations: $:^{38,39}$ refer to Sec. 2 in the Supplementary Material. 
a fluorescent dye is formed on a highly reflective mirror corresponding to the fluorescent emission wavelength. The volumes of droplets were fixed using a commercial inkjet printer (Fig. S2 in the Supplementary Material). Owing to the strong reflections of the mirror at two ends of the droplet and the internal reflections along the droplet-air interface between the two ends, a unique oscillation pathway of AL modes is formed [Fig. 1(a)]. An active layer of molecules is deposited at the interface between the droplet and mirror, where the hydrophobic forces govern the cavity geometry of a droplet laser [Fig. 1(b)]. Explicitly, the contact angle $\alpha$ that characterizes the cavity geometry is the result of balance among the droplet-solid interfacial tension $\gamma_{i}$, solid surface tension $\gamma_{s}$, and droplet surface tension $\gamma_{l}$. In principle, the AL modes that follow the oscillation paths corresponding to segments of " $N$-sided polygons" are highly correlated with the contact angles of droplet lasers. As the contact angle increases, AL modes with higher orders of $N$-sided polygon paths can be supported in the droplet, as shown in Fig. 1(c). The corresponding electric field distributions are simulated in Fig. 1(d) under different $N$. Consequently, more $\mathrm{AL}$ modes can be excited with the increase of contact angles, resulting in increased laser intensities.

\subsection{Demonstration of Lasing Action}

To demonstrate that "AL mode oscillations" are the result of strong reflections between the mirror and the droplet-air interface, two types of bottom substrates with different reflectivity and identical hydrophobicity (Fig. S3 in the Supplementary Material) were prepared. As compared in Figs. 2(a) and 2(c), FITC water/glycerol droplets were prepared on a glass slide (reflection $R<1 \%$ ) and a highly reflective DBR mirror ( $R>99.99 \%$ at FITC emission wavelength). Without highly reflective mirrors, no lasing mode was observed in the CCD image of the optically pumped droplet [inset in Fig. 2(b)], indicating no laser oscillations in the droplet. Similarly, no lasing spectrum was seen in Fig. 2(b); only a typical broad fluorescence emission profile was present. In contrast, lasing was observed under the same pump energy density with the addition of a highly reflective mirror. Strong laser modes were symmetrically emitted from the two opposite ends of the droplet [inset in Fig. 2(d)]. The corresponding emission spectrum shows typical lasing peaks of microlasers [Fig. 2(d)], which further indicates laser oscillations in the droplet. The results imply that the reflectivity of the bottom mirror substrate plays a vital role in the oscillations of droplet laser resonators.

To further demonstrate that internal reflections along the droplet-air interface also participate in laser oscillations, a "deformed" droplet was formed on a highly reflective mirror [Fig. 2(e)]. It is noteworthy that the random-shaped configuration definitely cannot support WGM that oscillates in the droplet bottom plane. Surprisingly, laser emissions were also observed under the same pump conditions [Fig. 2(f)]. Similar to the laser emission from a regular droplet resonator [inset in Fig. 2(d)], the laser signals were also emitted symmetrically from the two ends of the droplet [inset in Fig. 2(f)]. This finding indicates that internal reflections along the droplet surface between the two ends still participated in the laser oscillations, forming the proposed vertically orientated oscillation paths.

We further measured the output intensities and linewidths of a droplet resonator by increasing the pump energy densities to demonstrate lasing action. Spectrally integrated output intensities and linewidths were subsequently measured based on the spectra in Fig. 2(g), where the linewidth of a peak is defined by its full width at half-maximum [Fig. 2(h)]. As shown in Fig. 2(i), a clear threshold was observed for both the output intensities and linewidths. For instance, a relatively broader linewidth was measured when the pump energy density is below the threshold [Fig. 2(h)], which is contributed by enhanced spontaneous emissions. In contrast, linewidth narrowing was obtained when the pump energy density reached above the lasing threshold.

To investigate the properties of a laser output beam, an imaging spectrometer was used to observe the corresponding laser modes. As presented in Fig. S4 in the Supplementary Material, distinctive transverse modes with symmetrical sector shapes and nodal lines were observed from both ends of the microdroplet. The laser beams were found to possess a strong lasing polarization that is perpendicular to the direction along two opposite ends, indicating TE mode lasing in a droplet. Additionally, we investigated how different dye concentrations influence the lasing performance. Under a fixed contact angle, the output laser intensities increase with the increase of dye concentrations; meanwhile, the lasing thresholds decrease (Fig. S5 in the Supplementary Material). We further calculated the lasing thresholds by solving rate equations (Sec. 1 in the Supplementary Material). The calculation results have a good agreement with the experiment, which indicate that the lasing thresholds are consistent with the magnitudes of the gain and resonator losses ${ }^{40,41}$ [Sec. 1, Fig. S5(b) in the Supplementary Material].

\subsection{Characterization of Arc-Like Modes in Droplet Resonators}

Considering the fact that all the microdroplets used in this study are smaller than the capillary length, all the droplets can be regarded as parts of ideal spheres ${ }^{42-44}$ (Sec. 2 in the Supplementary Material). As such, it can support a series of laser oscillation modes propagating along pathways representing segments of $N$-sided polygons, as shown in Figs. 1(c) and 1(d). By following such pathways, the laser can be efficiently confined through the strong vertical reflections by the mirror and near-total reflections along the droplet-air interface. Considering the geometric characteristics of the AL oscillation paths, the number $N$ representing the side number of the $N$-sided polygon can be specified to characterize the AL mode. Determined by the axial symmetry of the AL oscillation path, $N$ is an even number. In practice, the AL oscillation paths cannot be directly observed due to a lack of scatterings in droplets. Nonetheless, AL modes with a specific $N$ can still be characterized by measuring their free spectrum ranges (FSRs). In a droplet resonator with a diameter of $D$ and a contact angle of $\alpha$ [Fig. 3(a)], the optical path length of the AL mode with a specific $N$ in a round trip can be calculated as (Sec. 3, Fig. S6 in the Supplementary Material)

$$
\begin{aligned}
L & =2 n[(N / 2-1) l+2 h] \\
& =\left[\frac{N \sin (\pi / N)-2 \cos \alpha}{\sin \alpha}\right] n D \quad\left(\frac{N-2}{2 N} \pi=\beta<\alpha\right),
\end{aligned}
$$

where $L$ is the optical path length of laser oscillation in a round trip and $n$ is the effective refractive index of droplet resonators. As shown in Fig. 3(a), $l$ is the side length of an $N$-sided polygon and $h$ is the length of two vertical short sides. It is worthy to note 
that the angle $\beta$ marked in Fig. 3(a) must be smaller than the contact angle $\alpha$, only in the condition wherein the oscillation path with $N$ can be permitted. Considering that the volume ratio of water/glycerol of droplets is $1: 1$, the refractive index was estimated to be $1.41 .^{44}$ As such, the corresponding FSR of AL mode with a specific $N$ can be defined as

$\mathrm{FSR}=c / L=\frac{c \cdot \sin \alpha}{[N \sin (\pi / N)-2 \cos \alpha] n D} \quad\left(\frac{N-2}{2 N} \pi=\beta<\alpha\right)$,

where $c$ is the speed of light. According to Eq. (2), we calculated the FSRs of AL modes as a function of contact angle [color lines in Fig. 3(b)]. If WGM oscillating along the bottom circle of a droplet is considered, the FSR will be $c / n \pi D$, which is independent of contact angles [black line in Fig. 3(b)]. As a comparison, we experimentally measured the FSRs of several lasing droplets with different contact angles [blue triangles in Fig. 3(b)] and found that the experimental data points fit well with the FSR of the AL modes.

To be more explicit, we demonstrate the lasing profiles of three individual droplet resonators with different contact angles (69 deg, $79 \mathrm{deg}$, and $85 \mathrm{deg}$ ). As shown in Figs. 3(c)-3(e), lasing spectra from the three droplets presented unique sets of FSRs as well as different numbers of AL modes. For instance, only one set of FSRs can be found in the droplet with a contact angle of $69 \mathrm{deg}$. According to Eq. (2), the calculated FSR corresponds to the AL mode with $N=4$ [Fig. 3(c)], while the simulated electric field with $N=4$ is also shown in Fig. 3(f). As the contact angle increased to $79 \mathrm{deg}$, two sets of FSRs corresponding to the AL modes with $N=4$ and $N=6$ were observed [Fig. 3(d)]. The simulated electric fields also support the AL modes with $N=4$ and $N=6$ [Fig. 3(g)]. For an even larger contact angle (85 deg), complicated lasing peaks were observed, including four sets of FSRs corresponding to $\mathrm{AL}$ modes with $N=6$, $N=8, N=10$, and $N=20$, respectively [Fig. 3(e)]. The simulated electric fields with $N=6, N=8, N=10$, and $N=20$ are also presented in Fig. 3(h), respectively. As such, the lasing spectra shown in Figs. 3(c)-3(e) present the tendency that more AL laser modes can be excited with the increase of contact angles.

\subsection{Lasing Action under Different Hydrophobic Forces}

Next, we attempt to investigate how hydrophobic forces interplay with the droplet resonator and influence the lasing output. For this purpose, mirrors with different surface hydrophobicity were prepared. The interfacial tension increases with the increasing hydrophobic forces at the droplet-solid interface, resulting in the increase of droplet contact angles. The resultant side-view images of the droplet lasers with various contact angles (58 deg to $90 \mathrm{deg}$ ) are shown in Fig. 4(a) (top row). Under the same FITC dye concentration and pump energy density, the lasing actions of the droplet lasers were studied as a function of interfacial tension as well as contact angle. The laser modes for the respective contact angles were recorded with a CCD in Fig. 4(a) (bottom row). At a relatively small contact angle (58 deg), no laser emission was observed. When the contact angle reached $67 \mathrm{deg}$, sharp laser emissions started to emerge. As the contact angles continued to rise, more intense laser modes with broader emission regions were observed. The statistics of spectrally integrated laser output intensities based on different interfacial tensions as well as contact angles are compared in Fig. 4(b). With the increase of interfacial tension and contact angles, a significant enhancement of laser output intensities was obtained, as shown in Fig. 4(b). The spectrally integrated laser outputs as a function of pump energy density under different contact angles were also investigated [inset in Fig. 4(b)], showing lower pump thresholds and higher slope efficiencies with the increase of interfacial tensions. Figure 4(c) shows the representative lasing spectra recorded under different interfacial tensions. The lasing intensity and number of lasing modes increased accordingly with the interfacial tension and contact angle, which can be attributed to an increased number $(N)$ of AL modes supported within the droplet. The linewidths of the lasing peaks are in the range of 0.13 to $0.2 \mathrm{~nm}$, which present a decreasing tendency as the contact angle increases [inset in Fig. 4(c)].

To understand the modulating mechanism, theoretical calculations of the $Q$-factor were carried out under different contact angles (based on fixed droplet volume). By increasing the contact angles from $58 \mathrm{deg}$ to $90 \mathrm{deg}$, a dramatic increase of $Q$-factors from $\sim 10^{3}$ to $10^{7}$ was achieved [red squares in Fig. 4(d)]. Note that under a specific contact angle, AL modes with different $N$ possess different $Q$-factors. However, only the maximum calculated $Q$-factors are plotted here in order to reflect the maximum capability of laser confinement. The maximum number of AL oscillation paths in a droplet that follow segments of $N$-sided polygons was also calculated based on reflection law (Sec. 3 in the Supplementary Material). For example, only AL oscillation paths that follow 4-sided and 6-sided polygons can be hosted in a droplet when the contact angle is fixed at $67 \mathrm{deg}$; therefore, the corresponding maximum number of AL oscillation paths is 2. As shown in Fig. 4(d), the blue line shows a significant increase of the maximum number of AL oscillation paths with the increasing contact angles. Based on the above theoretical results, the higher $Q$-factors at larger contact angles indicate lower optical losses. Additionally, the increasing maximum number of AL oscillation paths will result in more AL modes excited at larger contact angles. These two key factors co-determine the laser output intensities as well as lasing mode profiles as the contact angle changes. We also calculated the $Q$-factors of AL modes with different $N$ values under the same contact angle. In general, the AL mode with a larger $N$ possesses a higher $Q$-factor under the same contact angle [Fig. 4(e)]. The results are reasonable because the AL mode with a larger $N$ possesses larger internal reflection angles on the droplet-air interface, resulting in a lower loss and a higher $Q$-factor. Therefore, the AL laser modes with a larger $N$ and higher $Q$-factors will be excited as the contact angle increases [Fig. 4(f)].

\subsection{Modulating Laser Emission with Intermolecular Forces at the Biointerface}

Based on the above experimental and theoretical findings, we have explicitly demonstrated the correlation between laser emission intensities and hydrophobic forces at the droplet-solid interface. Herein, we investigated the possibility of modulating laser emissions of droplet resonators at the biointerface with different hydrophobic forces. Biointerface refers to the region where a biomolecule contacts an inorganic/organic surface, providing the basis for studying biomolecular interactions. ${ }^{28,45-48}$ 
As such, different concentrations of protein and peptide solutions were prepared in droplet resonators via inkjet printing in which biointerfaces were formed by biomolecular adsorptions on mirrors and the interfacial hydrophobicity was altered by biomolecular concentrations. The laser emission intensities of droplet resonators were subsequently recorded, as shown in Fig. 5(a). In the process of interface adsorption, non-polar side chains of biomolecules tend to be adsorbed on the hydrophobic surface, forming an interface with a higher hydrophilicity. ${ }^{49}$ The higher concentration of biomolecules adsorbed on the mirror surface, the lower the interfacial tension and contact angle will become. We first performed the statistics of interfacial tensions, contact angles, and laser output intensities as a function of BSA concentration. As shown in Fig. 5(b), the interfacial tension and contact angle decrease with the increase of BSA concentration, indicating a decrease of interfacial hydrophobicity. Meanwhile, the laser output intensities also decrease with the increase of BSA concentration. In particular, the hydrophobicity change induced by a $15 \mathrm{nM}$ concentration of BSA could be identified through droplet lasers. In addition to BSA, insulin and V5 peptide with different concentrations were also employed to modulate laser emissions of droplet resonators by tuning the interfacial hydrophobicity [Figs. 5(c) and 5(d)]. Figure 5 shows the significant changes in laser intensity under different molecular hydrophobicity (or molecular concentrations), showcasing the potential of modulating laser emissions with tiny interfacial molecular forces and hydrophobic interactions at the biointerface.

\section{Discussion and Conclusion}

In this work, we discovered a vertically orientated oscillation mode, AL mode, in mirror-supported droplets. In principle, WGM that oscillates horizontally along the droplet bottom circle can also be supported in such droplet resonators. ${ }^{14,15}$ However, when compared with WGM, AL modes were easier to oscillate in most experiments. To explore the reason for such a phenomenon, we compared the $Q$-factors of $\mathrm{AL}$ modes and WGM based on DBR mirror-supported droplet resonators with different contact angles (Fig. S7 in the Supplementary Material). As presented in Fig. S7(a) in the Supplementary Material, the $Q$-factors of AL modes are close to those of WGM under small contact angles $(<75 \mathrm{deg})$. However, the $Q$-factors become higher under larger contact angles $(>75 \mathrm{deg})$. The calculation results also indicate that $\mathrm{AL}$ modes can be better confined in droplets at large contact angles (75 deg to $90 \mathrm{deg}$ ) due to high reflections from DBR mirrors and droplet surfaces. In contrary, WGM resonators experience more losses during oscillation since a part of the energy will be coupled into DBR layers beneath [Fig. S7(b) in the Supplementary Material]. As a consequence, AL modes are relatively easy to be excited at large contact angles. Under small contact angles, the $Q$-factors of AL modes are slightly smaller than that of WGM. Nonetheless, when considering the unique distribution of the excitation light resonating along the droplet-air interface (Fig. S8 in the Supplementary Material), AL modes have the capability to cover much more gains than WGM in a droplet. Hence, AL modes can still be preferentially selected to oscillate under small contact angles. In fact, by decreasing the reflectivity of the supporting mirror at emission wavelengths, WGM can also be excited, presuming the increased loss of AL modes (Fig. S9 in the Supplementary Material). We also compared the $Q$-factors of
AL modes and WGM under contact angles above 90 deg (Fig. S10 in the Supplementary Material). For a contact angle that is slightly over $90 \mathrm{deg}$, the $Q$-factors of $\mathrm{AL}$ modes are still larger than that of WGM; however, they decrease and become much smaller than the $Q$-factors of WGM as the contact angle approaches $100 \mathrm{deg}$. When the contact angle is far above $90 \mathrm{deg}$, the $Q$-factors of AL modes decrease dramatically; while the $Q$-factors of WGM continue to increase. In such a case, WGM will dominate the oscillations in droplets. It is also noteworthy to point out that levitating droplets with optical tweezers can admit a $Q$-factor as high as $10^{9}$, which is 2 orders of magnitude higher than the $Q$-factor of a mirror-supported droplet. $^{50}$

One peculiar aspect of AL modes is the high correlation with contact angles, as compared to most WGMs. According to the calculation results shown in Fig. S7 in the Supplementary Material, the $Q$-factors of AL modes cover a much larger range $\left(10^{3}\right.$ to $\left.10^{7}\right)$ than that of WGM $\left(10^{3}\right.$ to $\left.10^{4}\right)$ as the contact angle increases. In addition, the max number of AL oscillation paths also increases dramatically with the increase of the contact angle [Fig. 4(d)]. In contrast, the only oscillation path of WGM is the droplet bottom circle, which is independent of the contact angle. Therefore, AL modes are much more sensitive to the interface hydrophobicity than WGM. It should be mentioned that the $Q$-factors of AL modes also increase with the increase of the droplet diameter [Fig. S11(a) in the Supplementary Material]. Under the contact angle of $90 \mathrm{deg}$, the $Q$-factors of a droplet resonator increase from $10^{4}$ to $10^{7}$ in a diameter range of 5 to $60 \mu \mathrm{m}$. In the current setup, the smallest droplet that can support lasing action with AL modes is approximately $5.7 \mu \mathrm{m}$ in diameter [Fig. S11(b) in the Supplementary Material].

In conclusion, we have revealed the underlying optical mechanism when a droplet resonator interacts with an interface and discovered its correlation with hydrophobic forces. A new laser oscillation mode-AL mode-was demonstrated in microdroplets. Both experimental and theoretical investigations verified that AL lasing modes are strongly modulated by the hydrophobic forces at droplet-solid interfaces. The number of excited AL modes and $Q$-factors increases as the hydrophobic forces (interfacial tension and contact angle) increase, resulting in a significant enhancement of laser intensity. As a proof-ofconcept, biointerfaces induced by adsorptions of protein and peptide on mirrors were employed to modulate laser emissions of droplet resonators. The output laser intensities were also strongly modulated by intermolecular forces under various biomolecular concentrations. Our findings demonstrate that laser emission has the potential to amplify subtle molecular force changes, paving the way for studying biomechanics and biophysics at the interface.

\section{Acknowledgments}

We would like to thank the lab support from Centre of BioDevices and Bioinformatics and Internal Grant NAP SUGM4082308.040 from Nanyang Technological University. The authors declare no conflicts of interest.

\section{References}

1. T. Reynolds et al., "Fluorescent and lasing whispering gallery mode microresonators for sensing applications," Laser Photonics Rev. 11(2), 1600265 (2017). 
2. U. Bog et al., "On-chip microlasers for biomolecular detection via highly localized deposition of a multifunctional phospholipid ink," Lab Chip 13(14), 2701-2707 (2013).

3. Y. F. Zhang et al., "Tunable microlasers modulated by intracavity spherical confinement with chiral liquid crystal," Adv. Opt. Mater. 8(10), 1902184 (2020).

4. Y.-C. Chen et al., "Laser-emission imaging of nuclear biomarkers for high-contrast cancer screening and immunodiagnosis," Nat. Biomed. Eng. 3(9), 724-735 (2017).

5. E. Ignesti et al., "A new class of optical sensors: a random laser based device," Sci. Rep. 6(1), 35225 (2016).

6. S. Nizamoglu et al., "A simple approach to biological single-cell lasers via intracellular dyes," Adv. Opt. Mater. 3(9), 1197-1200 (2015).

7. Q. Chen et al., "An integrated microwell array platform for cell lasing analysis," Lab Chip 17(16), 2814-2820 (2017).

8. Z. Yuan et al., "Lasing-encoded microsensor driven by interfacial cavity resonance energy transfer," Adv. Opt. Mater. 8(7), 1901596 (2020).

9. Y. C. Chen and X. Fan, "Biological lasers for biomedical applications," Adv. Opt. Mater. 7(17), 1900377 (2019).

10. H. Azzouz et al., "Levitated droplet dye laser," Opt. Express 14(10), 4374-4379 (2006).

11. D. McGloin, "Droplet lasers: a review of current progress," Rep. Prog. Phys. 80(5), 054402 (2017).

12. A. Jonáš et al., "In vitro and in vivo biolasing of fluorescent proteins suspended in liquid microdroplet cavities," Lab Chip 14(16), 3093-3100 (2014).

13. S. Nizamoglu et al., "All-biomaterial laser using vitamin and biopolymers," Adv. Mater. 25(41), 5943-5947 (2013).

14. V. D. Ta et al., "Multicolor lasing prints," Appl. Phys. Lett. 107(22), 221103 (2015)

15. J. Zhao et al., "Full-color laser displays based on organic printed microlaser arrays," Nat. Commun. 10(1), 870 (2019).

16. T. V. Nguyen and V. D. Ta, "High-quality factor, biological microsphere and microhemisphere lasers fabricated by a single solution process," Opt. Commun. 465, 125647 (2020).

17. Z. Zhou et al., "Organic printed core-shell heterostructure arrays: a universal approach to all-color laser display panels," Angew. Chem. Int. Ed. 59(29), 11814-11818 (2020).

18. P. A. Alekseev et al., "Half-disk laser: insight into the internal mode structure of laser resonators," Opt. Express 26(11), 14433-14443 (2018).

19. D. Leckband and J. Israelachvili, "Intermolecular forces in biology," Q. Rev. Biophys. 34(2), 105-267 (2001).

20. P. Ball, "Water is an active matrix of life for cell and molecular biology," Proc. Natl. Acad. Sci. USA 114(51), 13327-13335 (2017).

21. K. A. Dill and J. L. MacCallum, "The protein-folding problem, 50 years on," Science 338(6110), 1042-1046 (2012).

22. A. Hinderliter and S. May, "Cooperative adsorption of proteins onto lipid membranes," J. Phys. Condens. Mater. 18(28), S1257-S1270 (2006).

23. A. Rimola et al., "Silica surface features and their role in the adsorption of biomolecules: computational modeling and experiments," Chem. Rev. 113(6), 4216-4313 (2013).

24. Y. Yan et al., "Albumin adsorption on CoCrMo alloy surfaces," Sci. Rep. 5(1), 18403 (2016).

25. M. J. Limo et al., "Interactions between metal oxides and biomolecules: from fundamental understanding to applications," Chem. Rev. 118(22), 11118-11193 (2018).

26. D. Costa et al., "Understanding small biomolecule-biomaterial interactions: a review of fundamental theoretical and experimental approaches for biomolecule interactions with inorganic surfaces," J. Biomed. Mater. Res. A 101(4), 1210-1222 (2013).

27. C. Mathe et al., "Structural determinants for protein adsorption/nonadsorption to silica surface," PLOS ONE 8(11), e81346 (2013).

28. A. Sun and J. Lahann, "Dynamically switchable biointerfaces," Soft Matter 5(8), 1555-1561 (2009).
29. I. T. S. Li and G. C. Walker, "Signature of hydrophobic hydration in a single polymer," Proc. Natl. Acad. Sci. USA 108(40), 1652716532 (2011).

30. J. Kim et al., "A dynamic hydrophobic core orchestrates allostery in protein kinases," Sci. Adv. 3(4), e1600663 (2017).

31. S. A. Harris and V. M. Kendon, "Quantum-assisted biomolecular modelling," Philos. T. R. Soc. A 368(1924), 3581-3592 (2010).

32. A. Kiraz et al., "Spectral tuning of liquid microdroplets standing on a superhydrophobic surface using electrowetting," Appl. Phys. Lett. 92(19), 191104 (2008).

33. A. Jonas et al., "Probing microscopic wetting properties of superhydrophobic surfaces by vibrated micrometer-sized droplets," Langmuir 27(6), 2150-2154 (2011).

34. D. Cwikel et al., "Comparing contact angle measurements and surface tension assessments of solid surfaces," Langmuir 26(19), 15289-15294 (2010).

35. D. Y. Kwok and A. W. Neumann, "Contact angle interpretation in terms of solid surface tension," Colloids Surf. A 161(1), 31-48 (2000).

36. D. Khossravi and K. A. Connors, "Solvent effects on chemical processes. 3. Surface-tension of binary aqueous organic-solvents," J. Solution Chem. 22(4), 321-330 (1993).

37. X. X. Lin et al., "Influence of water evaporation/absorption on the stability of glycerol-water marbles," RSC Adv. 9(59), 3446534471 (2019).

38. A. T. Palasz et al., "The effect of macromolecular supplementation on the surface tension of TCM-199 and the utilization of growth factors by bovine oocytes and embryos in culture," Animal Reprod. Sci. 58(3-4), 229-240 (2000).

39. I. D. Kamalanathan and P. J. Martin, "Competitive adsorption of surfactant-protein mixtures in a continuous stripping mode foam fractionation column," Chem. Eng. Sci. 146, 291-301 (2016).

40. Y.-C. Chen et al., "Versatile tissue lasers based on high- $Q$ FabryPérot microcavities," Lab Chip 17(3), 538-548 (2017).

41. M. Aas et al., "Optofluidic FRET lasers and their applications in novel photonic devices and biochemical sensing," IEEE J. Sel. Top. Quantum Electron. 22(4), 7000215 (2016).

42. J. Chatterjee, "Limiting conditions for applying the spherical section assumption in contact angle estimation," J. Colloid Interface Sci. 259(1), 139-147 (2003).

43. M. Mastrangeli et al., "Surface tension-driven self-alignment," Soft Matter 13(2), 304-327 (2017).

44. K. Takamura et al., "Physical properties of aqueous glycerol solutions," J. Petrol. Sci. Eng 98-99, 50-60 (2012).

45. O. Parlak and A. P. F. Turner, "Switchable bioelectronics," Biosens. Bioelectron. 76(SI), 251-265 (2016).

46. Y. Zhou et al., "Multistimulus responsive biointerfaces with switchable bioadhesion and surface functions," ACS Appl. Mater. Inter. 12(5), 5447-5455 (2020).

47. D. Chandler, "Interfaces and the driving force of hydrophobic assembly," Nature 437(7059), 640-647 (2005).

48. C. Q. Zhu et al., "Characterizing hydrophobicity of amino acid side chains in a protein environment via measuring contact angle of a water nanodroplet on planar peptide network," Proc. Natl. Acad. Sci. USA 113(46), 12946-12951 (2016).

49. M. Rabe et al., "Understanding protein adsorption phenomena at solid surfaces," Adv. Colloid Interface Sci. 162(1-2), 87-106 (2011).

50. J. Kher-Alden et al., "Microspheres with atomic-scale tolerances generate hyperdegeneracy," Phys. Rev. X 10(3), 031049 (2020).

Zhen Qiao is a research fellow at Nanyang Technological University, Singapore.

Xuerui Gong is a PhD graduate student at Nanyang Technological University, Singapore. 
Peng Guan is a master graduate student at Shanghai Jiao Tong University, China.

Zhiyi Yuan is a PhD graduate student at Nanyang Technological University, Singapore.

Shilun Feng is currently an associate professor at Shanghai Institute of Microsystem and Information Technology, Chinese Academy of Sciences, China. His research focuses on microfluidics and devices for precision medicine.

Yiyu Zhang is a research fellow at Nanyang Technological University, Singapore.
Munho Kim is currently an assistant professor at Nanyang Technological University, Singapore.

Guo-En Chang is a professor at National Chung Cheng University, Taiwan.

Yu-Cheng Chen received a $\mathrm{PhD}$ in biomedical engineering from the University of Michigan, Ann Arbor, in 2017 and has worked as an assistant professor at Nanyang Technological University, Singapore, since 2018. His research mainly focuses on optofluidics, biolasers, biophotonics, biosensors and imaging, and novel on-chip laser devices. 